Letter to the Editor

\title{
Factors affecting keratoconus progression and corneal collagen cross-linking
}

\author{
Elsie Chan FRANZCO, ${ }^{1,2}$ Harry Yip MBBS, ${ }^{1}$ Sara Vogrin MBiostat ${ }^{3,4}$ and Srujana \\ Sahebjada $\mathrm{PhD}^{2,3}$
}

1. Royal Victorian Eye and Ear Hospital, Melbourne, Australia

2. Centre for Eye Research Australia, University of Melbourne, Melbourne Australia

3. University of Melbourne, Melbourne, Australia

4. St Vincent's Hospital, Melbourne, Australia

Correspondence: Elsie Chan, Royal Victorian Eye and Ear Hospital, 32 Gisborne St, East Melbourne, VIC 3002, Australia

Email: elsie.chan@eyeandear.org.au

Received 17 J anuary 2020; accepted 25 J anuary 2020

Funding sources / Financial disclosure: None

Conflict of interest: None

This is the author manuscript accepted for publication and has undergone full peer review but has not been through the copyediting, typesetting, pagination and proofreading process, which may lead to differences between this version and the Version of Record. Please cite this article as doi: 10.1111/ceo.13723

This article is protected by copyright. All rights reserved. 
Keratoconus is a corneal ectasia where progression can lead to decreased visual acuity and significant morbidity. Corneal cross-linking (CXL) may halt the disease in cases of worsening keratoconus, however, there is no universal definition for disease progression $^{1}$. It is also not known what factors influence the success of CXL, although some studies report that K2 (mean keratometry on the steepest axis) greater than 54 dioptres $^{2}$, age between 18 and 39 years old ${ }^{3}$ and a central cone ${ }^{4}$ are associated with greater flattening following treatment. One factor that has not been investigated is whether the degree of pre-CXL progression affects post-CXL outcomes. It is also unclear if CXL should be offered on first presentation to individuals who have more risk factors for progression.

We performed a retrospective study on patients with progressive keratoconus who underwent CXL at the Royal Victorian Eye and Ear Hospital, Melbourne, Australia between J uly 2007 and February 2016. The aims of this study were to 1) investigate the preoperative factors that may influence the outcomes after $\mathrm{CXL} ; 2$ ) to determine whether the rate of pre-operative progression affects treatment outcomes; 3 ) examine the percentage of fellow eyes which were treated within the follow-up period. Only patients who had CXL at our institution with at least 2 pre-operative visits, and follow-up of at least 11 months after CXL were included in the study.

CXL in each patient was performed using the epithelium-off, Dresden protocol. Epithelium was removed manually with a blade or following $20 \%$ alcohol for 20 seconds. Riboflavin $0.1 \%$ (with $20 \%$ dextran) was applied topically for 30 minutes and ultraviolet $A$ irradiation (370nm) was delivered for 30 minutes at $3 \mathrm{~mW} / \mathrm{cm}^{2}$. The corneal thickness was measured every 10 minutes; if the thickness was below $400 \mu \mathrm{m}$, hypotonic riboflavin was used until the thickness was above $400 \mu \mathrm{m}$. Data was analysed between the baseline (visit 1) and immediate pre-operative visit (visit 2), and with the 11 month post-operative visit (visit 3). 
A total of 110 patients were included in this study. The baseline characteristics shown in Table 1 . Visit 1 was a mean of 8 months prior to CXL, visit 2 was two months prior to $C X L$, and visit 3 was a mean of 14 months after $C X L$.

Table 1: Patient characteristics. K1, K2 and Kmax were measured using the Pentacam (Oculus, Germany).

\begin{tabular}{|c|c|}
\hline & $\begin{array}{l}\text { Median (IQR) / N } \\
(\%)\end{array}$ \\
\hline Total & $110(100 \%)$ \\
\hline Age & $\begin{array}{l}21(18,26), \text { range } 11- \\
53\end{array}$ \\
\hline Sex (male) & $69(63 \%)$ \\
\hline $\begin{array}{l}\text { Baseline parameters } \\
\qquad \begin{array}{l}\text { K1 } \\
\text { K2 } \\
\text { Kmax } \\
\text { Thinnest corneal thickness } \\
\text { Refractive sphere } \\
\text { Refractive cylinder }\end{array} \\
\end{array}$ & $\begin{array}{l}47.6(45,51.2) \\
53.1(49.7,57.1) \\
60.6(56.5,66.8) \\
436(413,460) \\
1.5(-0.5,3) \\
-4.5(-7,-2.8) \\
\end{array}$ \\
\hline $\begin{array}{l}\text { Outcome of fellow eye during follow-up } \\
\text { period } \\
\qquad \begin{array}{l}\text { Corneal Graft } \\
\text { CXL } \\
\text { Advanced keratoconus } \\
\text { (unsuitable for CXL) } \\
\text { Stable keratoconus }\end{array}\end{array}$ & $\begin{array}{l}9(9 \%) \\
53(48 \%) \\
4(4 \%) \\
44(40 \%)\end{array}$ \\
\hline
\end{tabular}

Table 2: Changes in outcome parameters between visits.

\begin{tabular}{|l|l|l|l|l|}
\hline & Visit 1-2 & Visit 2-3 \\
\hline & Coefficient, 95\% Cl & $p$ & $\begin{array}{l}\text { Coefficient, 95\% } \\
\text { Cl }\end{array}$ & $p$ \\
\hline K1 & $1.34(0.65,2.04)$ & $<0.001$ & $0.51(0.11,0.90)$ & 0.011 \\
\hline
\end{tabular}




\begin{tabular}{|l|l|l|l|l|}
\hline K2 & $1.96(1.23,2.68)$ & $<0.001$ & $0.40(-0.01,0.81)$ & 0.054 \\
\hline Kmax & $2.04(0.85,3.22)$ & 0.001 & $0.36(-0.30,1.03)$ & 0.284 \\
\hline $\begin{array}{l}\text { Corneal } \\
\text { thickness }\end{array}$ & $\begin{array}{l}-10.34(-18.57,- \\
2.10)\end{array}$ & 0.049 & $-13(-18,-8)$ & $<0.001$ \\
\hline Sphere & $0.45(-0.65,-1.56)$ & 0.422 & $0.13(-0.53,0.78)$ & 0.706 \\
\hline Cylinder & $-0.49(-0.52,1.50)$ & 0.343 & $\begin{array}{l}-0.70(-1.29,- \\
0.10)\end{array}$ & 0.022 \\
\hline
\end{tabular}

Pre-operatively, there was a correlation between a younger age and greater steepening of $\mathrm{K} 1$ (average keratometry on the flatter axis) and $\mathrm{K} 2$ over the two visits prior to CXL (visits 1 and 2). There was also a moderate to strong correlation between a steeper baseline $K 2$ and progression in $K 2(r=0.608)$ and a steeper Kmax (maximum keratometry value) and progression in $K \max (r=0.616)$. Sex had no effect on K1, K2 or Kmax (Table 2).

Post-operatively, K1, K2, Kmax, corneal thickness at the thinnest point and cylinder on subjective refraction remained stable with no correlation with age, sex or rate of pre-CXL progression. Patients with a larger change in spherical error prior to surgery had a larger decrease in spherical error following CXL (interaction coefficient -0.01, $95 \% \mathrm{Cl}-0.02,-0.00, \mathrm{p}=0.010$ ) (Table 2 ).

The outcomes of the fellow eyes over the follow-up period are shown in Table 1. Forty-four (40\%) of eyes had stable keratoconus in the fellow eye. Four (4\%) had advanced keratoconus and were not eligible for CXL.

This retrospective study demonstrates that risk factors for pre-CXL progression are a younger age and steeper keratometry values, which is consistent with the findings of Ferdi et $a$. These results are important when counselling patients regarding the risk factors for pre-CXL progression and should influence the time interval between repeat examinations to detect progressive disease. However, the rate of progression 
prior to CXL did not affect the success of the treatment in halting the disease following CXL.

$40 \%$ of fellow eyes in this study had stable keratoconus over the follow-up period, which suggests that routinely treating fellow eyes before documenting progression would also lead to overtreatment. Thirteen percent of patients presented with advanced keratoconus in their fellow eye where CXL was no longer suitable or where a corneal transplant indicated, suggesting that 1 in 8 patients are still presenting to our clinic late in the course of the disease.

The limitations of this study are in its retrospective design. During the follow-up period, there was a change in the ultraviolet A device during this time, and there were different operators performing the $\mathrm{CXL}$ and assessing the patients pre- and post-CXL. A larger sample size would be required to validate these findings.

In conclusion, we found that a younger age and steeper keratometry values are risk factors for progression of keratoconus. The rate of pre-CXL progression did not affect treatment outcomes.

\section{REFERENCES}

1. Gomes J A, Tan D, Rapuano CJ et al, Group of Panelists for the Global Delphi Panel of K, Ectatic D. Global consensus on keratoconus and ectatic diseases. Cornea 2015; 34: 359-69.

2. Wittig-Silva C, Chan E, I slam FM, Wu T, Whiting M, Snibson GR. A randomized, controlled trial of corneal collagen cross-linking in progressive keratoconus: three-year results. Ophthalmology 2014; 121: 812-21. 
3. Vinciguerra R, Romano MR, Camesasca FI, et al. Corneal cross-linking as a treatment for keratoconus: four-year morphologic and clinical outcomes with respect to patient age. Ophthalmology 2013; 120: 908-16.

4. Godefrooij DA, Boom K, Soeters N, Imhof SM, Wisse RP. Predictors for treatment outcomes after corneal crosslinking for keratoconus: a validation study. Int Ophthalmo/ 2017; 37: 341-8.

5. Ferdi AC, Nguyen V, Gore DM, Allan BD, Rozema JJ, Watson SL. Keratoconus Natural Progression: A Systematic Review and Meta-analysis of 11529 Eyes. Ophthalmology 2019; 126: 935-45. 


\section{University Library}

\section{- M M N E R VA A gateway to Melbourne's research publications}

Minerva Access is the Institutional Repository of The University of Melbourne

Author/s:

Chan, E;Yip, H;Vogrin, S;Sahebjada, S

Title:

Factors affecting keratoconus progression and corneal collagen cross-linking

Date:

2020-02-17

Citation:

Chan, E., Yip, H., Vogrin, S. \& Sahebjada, S. (2020). Factors affecting keratoconus progression and corneal collagen cross-linking. CLINICAL AND EXPERIMENTAL OPHTHALMOLOGY, 48 (4), pp.531-532. https://doi.org/10.1111/ceo.13723.

Persistent Link:

http://hdl.handle.net/11343/275427 